\title{
Multi-needle Langmuir probe operation and acute probe current susceptibility to spacecraft potential
}

\author{
Magnus F. Ivarsen ${ }^{1}$, Huy Hoang ${ }^{1}$, Lei Yang ${ }^{1}$, Lasse B. N. Clausen ${ }^{1}$, Andres Spicher ${ }^{1}$, \\ Yaqi Jin $^{1}$, Espen Trondsen ${ }^{1}$, Jøran I. Moen ${ }^{1}$, Ketil Røed ${ }^{1}$, Bjørn Lybekk ${ }^{1}$
}

\begin{abstract}
NorSat-1 was launched on 14 July 2017 as a satellite carrying, among other instruments, the multi-Needle Langmuir Probe (m-NLP), an instrument which, on NorSat1 , is capable of measuring the ionospheric plasma electron density with the high sampling frequency of $1000 \mathrm{~Hz}$. The $m$-NLP instrument operates by analyzing the current-voltage diagram resulting from the measurements from each individual probe. In principle, the m-NLP operation methodology should be insensitive to spacecraft charging. However, this is not always the case. In this paper, we present an overview of the instrument response to passes into and out of eclipse. When the satellite exits eclipse, we observe a collapse in the collected probe currents. This acute drop is unaccounted for by the theoretical operation of the instrument. We present a statistical analysis of the phenomenon based on several months of NorSat-1 data, and we suggest a plausible reason for the observed drop in the current, namely spacecraft charging by solar cell arrays upon eclipse exit. We briefly discuss how satellite orientation and plasma wake affect the current drop. With this paper, we address Langmuir probe current susceptibility to spacecraft potential.
\end{abstract}

\section{INTRODUCTION}

The NorSat-1 satellite, a multi-payload micro-satellite weighing $15.6 \mathrm{~kg}$, was developed by Space Flight Laboratory (UTIAS-SFL) in Canada. Norway's first scientific satellite, it was launched with several objectives in mind. Firstly, the satellite is part of the government of Norway's effort to track ship traffic from space, using the Automatic Identification System (AIS), an objective shared by its twin satellite NorSat2, launched at the same time. The AIS system was built by Kongsberg Seatex in Norway. In addition to the AIS receiver used to monitoring maritime traffic, NorSat-1 carries two scientific instruments. The Compact Lightweight Absolute Radiometer (CLARA) measures total solar irradience in an effort to monitor solar activity [1]. CLARA was built by Physikalisch Meteorologisches Observatorium Davos / World Radiation Center in Switzerland. To make high-resolution measurements of the upper ionospheric plasma density, NorSat-1 was equipped with the multi-Needle Langmuir Probe system (m-NLP), designed by the Department of Physics at the University of Oslo, Norway [2], [3].

In Fig. 1, we present a schematic overview of NorSat1. Being a relatively small satellite, NorSat- 1 measures approximately $23 \mathrm{~cm} \times 39 \mathrm{~cm} \times 44 \mathrm{~cm}$, with a primarily aluminum structure. The main solar cell array and the CLARA instrument are mounted on one side of the body, while the

\footnotetext{
${ }^{1}$ Department of Physics, University of Oslo, Oslo, Norway

This research has received financial support from Research Council of Norway project 208006, 230996 and ESA PRODEX project 4200090335.
}

AIS is mounted on the opposite side. Smaller solar cell panels are placed on all sides of the satellite body. The m-NLP system protrudes from the body on booms mounted on the corner of the same side as the AIS, shown in the far $+z$ direction in Fig. 1. The satellite body is designed to expose maximal conducting metal surface to the environment to provide grounding to the electrical components. NorSat-1 has a total of approximately $3800 \mathrm{~cm}^{2}$ of conducting outer surface. The satellite's attitude control system is missionconfigurable, but usually operates to keep the solar cell arrays and CLARA pointed towards the Sun.

The Langmuir probes are placed on four booms of length $370 \mathrm{~mm}$. The probes are designed to be small enough to fit within the probe Debye shielding region in typical low Earth orbit plasma conditions. They have a diameter of 0.5 $\mathrm{mm}$ and are $25 \mathrm{~mm}$ long. In addition, each probe comes with a conductive bootstrapped section with a diameter of $2 \mathrm{~mm}$ and a length of $15 \mathrm{~mm}$. Probes and bootstraps are carboncoated, and both are held at the same bias. At the bottom of the bootstrap section, a small plastic base separates the probe and bootstrap from the conducting aluminum boom, which is coated with Alodine 1200 to improve conductivity. The length of the booms are constrained by the spacecraft frame size, as they deploy from the spacecraft. The booms, being deployed from the satellite after launch, were designed to protrude as far away from the satellite body as possible. However, depending on the satellite orientation, several

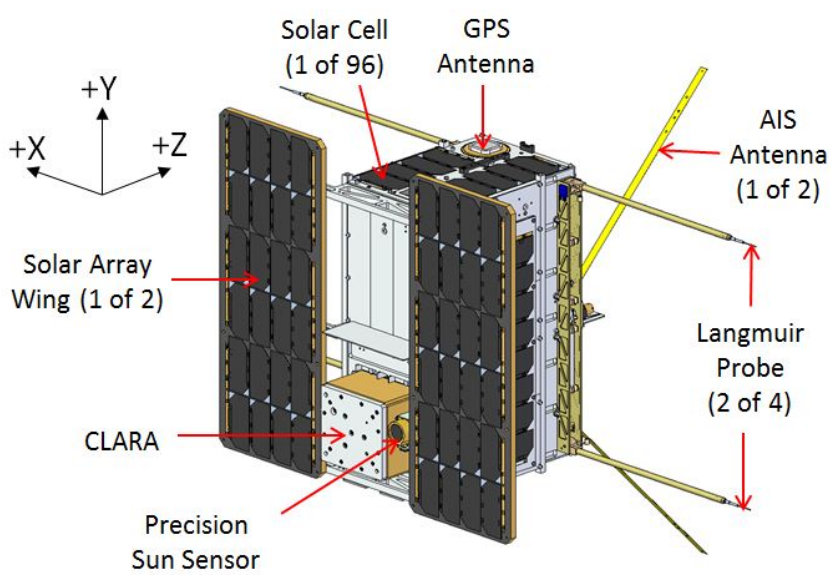

Fig. 1. Schematic drawing of the NorSat-1 satellite. $\mathrm{X}, \mathrm{Y}$ and $\mathrm{Z}$ directions represent the spacecraft coordinate system. The m-NLP probes are mounted on booms that deployed after launch, while the solar cell arrays were predeployed. Figure from SFL. 
probes might be situated inside the plasma wake.

NorSat-1 orbits Earth in a retrograde polar orbit with an inclination of 97.6 degrees, at approximately $600 \mathrm{~km}$ altitude, and in a virtually circular orbit with an eccentricity of 0.0014. Furthermore, the satellite is in a stable quasiheliosynchronous orbit. As such, the orbit with respect to the position of the Sun does not change significantly with time, as is seen from the orbit trace displayed in Fig. 3. NorSat-1 orbits from the dayside in the northern hemisphere through the equatorial nightside to the southern hemisphere, and enters the dayside again in the southern polar/sub-polar region.

\section{m-NLP operation}

The multi-Needle Langmuir Probe was developed at the University of Oslo [2], [3]. It was used on board the ICI-2 sounding rocket [4]-[6], and has since been used on several sounding rockets (see e.g. [7], [8]). The m-NLP system is also on-board 11 satellites of the European QB50 cubesat constellation [9].

Langmuir probes are commonly used to measure plasma characteristics such as electron density $n_{e}$ and temperature $T_{e}$, in addition to ion temperature $T_{i}$. A probe, when immersed in a plasma, collect current according to its electric charge, or bias. The collected current is described by orbital-motionlimited (OML) theory [10], which models charge carrier trajectories around spherical or cylindrical probes with radii much smaller than the plasma Debye length.

Langmuir probe operation normally consists of sweeping the probe through a range of voltages from negative to positive. Sweeping produces a curve such as the one shown in Fig. 2, from which the mentioned plasma parameters can be determined [11], [12]. However, a sweep typically takes around $1 \mathrm{~s}$ to perform [13]. The m-NLP system on board NorSat-1 is capable of measuring electron density with a temporal frequency of $1000 \mathrm{~Hz}$, or 1000 measurements per second. Such a high sampling frequency is accomplished by having four individual Langmuir probes biased at different voltages, from which the Langmuir I-V curve is estimated, given that the four probes collect current in the electron saturation regime (see Fig. 2). This method bypasses the comparatively long time required to perform a single sweep.

Within the conditions required by OML theory, the electron saturation current of a single probe is given by, [14], [15]

$$
I_{c}=N_{e} A e \frac{2}{\sqrt{\pi}} \sqrt{\frac{k_{B} T_{e}}{2 \pi m_{e}}}\left(1+\frac{e V}{k_{B} T_{e}}\right)^{\beta},
$$

where $N_{e}$ is the electron density, $A$ the probe area, $e$ the elementary charge, $k_{B}$ the Boltzmann constant, $T_{e}$ the electron temperature, and $m_{e}$ is the electron mass. $V$ is the probe bias potential $\left(V_{b}\right)$ with respect to the plasma potential $\left(V_{p}\right)$. That is, $V=V_{b}-V_{p}$. The exponent $\beta$ has the value 0.5 for ideal cylindrical Langmuir probes (see discussion below).

In order for Eq. (1) to be valid, the plasma needs to be non-drifting, collisionless, and non-magnetized. In the Fregion ionosphere, the thermal speed of electrons is larger than the spacecraft speed, the mean free path of electrons is

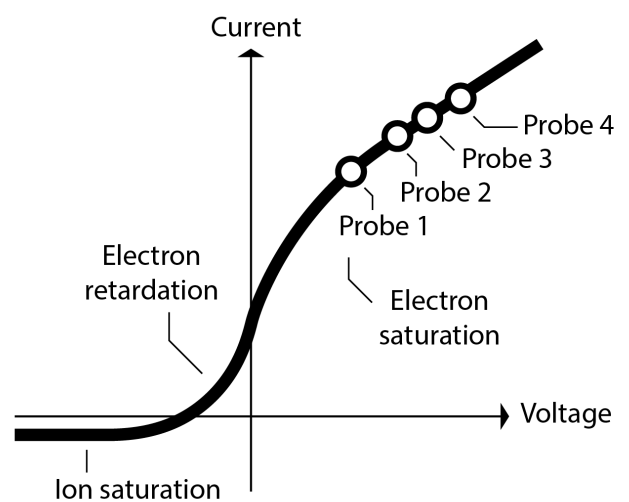

Fig. 2. Langmuir I-V curve for cylindrical probes. The four m-NLP system probes are marked on the curve in the electron saturation regime.

sufficiently long, and the Larmor radius is much larger than the probe radius. Thus, the mentioned conditions are fulfilled [2], [15]. The configurable probe bias, $V_{b}$, is relative to the electrical ground, which is the spacecraft chassis. The mNLP method of determining electron density then requires the difference in the square of collected currents $\Delta I_{c}^{2}$, and the difference in the probe biases $\Delta V_{b}$ :

$$
N_{e}=\frac{1}{K A} \sqrt{\frac{\Delta I_{c}^{2}}{\Delta V_{b}}}
$$

where $\beta$ from Eq. (1) is assumed to be 0.5 , and where,

$$
K=\frac{e^{3 / 2}}{\pi} \sqrt{\frac{2}{m_{e}}},
$$

is a constant factor based on the electron charge and mass.

There are in fact several reported problems arising in the application of OML theory to cylindrical Langmuir probe plasma measurements, and as such in using Eq. (2) to estimate plasma density. As mentioned, in OML theory, Langmuir probe charge collection is treated by considering the orbital motion of charge carriers around the probe, within the probe's electrostatic sheath. However, the effective absorption surface area as seen by a charge carrier varies, causing the charge carrier absorption rate to deviate from the theoretical prediction [16]. Further, the requirement that the plasma surrounding the probe be collisionless is likewise broken in many practical situations, leading to inapplicability of OML theory [16]. Measuring hot filament-generated laboratory plasma with a cylindrical Langmuir probe, [17] found that, in the electron saturation regime, the $\beta$-exponent in Eq. (1) can take on values up to 0.75 , deviating from the theorized value of 0.5 for cylindrical probes. The authors suggest that when the local plasma Debye length is sufficiently long, the probe radius becomes substantial, and the probe electrostatic sheath starts to resemble that of a spherical probe. Likewise using a hot filament generated plasma, [18] found that the $\beta$-exponent can, when the local plasma Debye length is sufficiently long, vary approximately between 0.4 


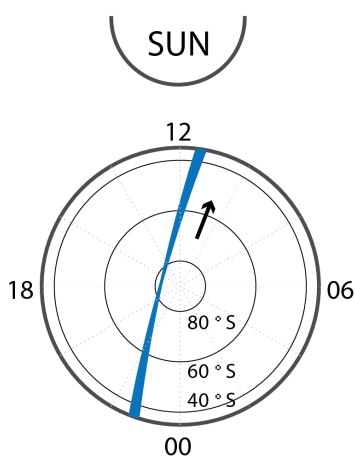

SOUTH

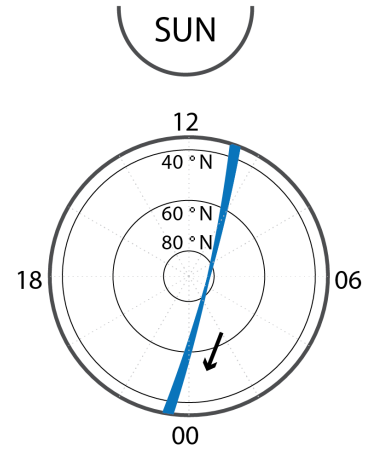

NORTH
Fig. 3. The trace made by NorSat-1's orbit as seen in a local solar time frame. The clock positions indicates local solar time, 12 corresponding to local noon, and 00 corresponding local midnight. The latitudinal degrees correspond to geographic latitude. The orbit trace data used consists of three months of operation.

and 0.9 . The authors suggest that a cylindrical probe should be theoretically modelled as a half-hemisphere attached to a cylinder, in order to correctly predict current collection. Theoretically, the electron density prediction (Eq. 2) from $\mathrm{m}$-NLP probe operation is sensitive to $\beta$-deviations, with a $20 \%$ increase in the value of $\beta$ causing a $45 \%$ deviation in estimated density [19].

Evidence from in-situ measurements on the accuracy of m-NLP probe density estimation (Eq. 2) exists. Analyzing data from the m-NLP system mounted on sounding rockets, [14] found that the estimate is within $30 \%$ of absolute measurements performed with a Faraday rotation experiment. Lastly, methods to correct for the inaccuracies of OML-theory to cylindrical Langmuir probe operation have been suggested [17]-[19]. Since the results reported in the present study do not directly depend on the accuracy of the m-NLP density prediction, we shall not further discuss issues related to $\mathrm{m}$ NLP probe operation.

\section{Solar cell arrays}

The power source for NorSat-1 is provided by 8 -cell $28 \%$ maximum efficiency solar cell arrays. The power system, which includes the main solar cell array, several bodyattached solar cell panels, and a 6-cell battery pack capable of providing 9.6 Ah, is grounded in the spacecraft body. When switched on, the solar cell circuit has a peak power voltage varying from approximately $23 \mathrm{~V}$ at $-50^{\circ} \mathrm{C}$ to approximately $16 \mathrm{~V}$ at $+85^{\circ} \mathrm{C}$. The cell units are organized in a grid with small gaps between each individual unit.

\section{Spacecraft charging}

Since the space age started, spacecraft charging has been a recognized issue and source for potential hazard in space [20], [21]. A spacecraft must reach electrical equilibrium with its plasma environment. By acquiring surface charges, the net electrical current between plasma and spacecraft becomes zero [21]. A spacecraft potential is then established with respect to the plasma environment. Several charging mechanisms exist, such as electron photo-emission by incident sunlight, changes in the surrounding electron density and the response of spacecraft conductive surfaces, and incident high-energy particle precipitation [21], [22]. Charging by solar cell arrays poses many challenges to spacecraft operation, both in the form of permanently damaging discharges called arcs [23], [24], and in the form of solar cell charge collection affecting the spacecraft potential [25], [26].

The rest of this article will be organized as follows. In Section II we will introduce the problem at hand. In Section III we will go through the data collected, and the statistical analysis performed on it. In Section IV we will present a plausible explanation for the probe current collapse, and discuss its implications. We provide concluding remarks in Section V.

\section{ACUTE CURRENT COLLAPSE}

Soon after the NorSat-1 launch, data showed that when the satellite exits eclipse, the current collected by the probes collapses. This current collapse is best illustrated by Fig. 4, which shows detailed NorSat-1 data from around 00:30 UT on 3 November 2017, when the probe biases were $6 \mathrm{~V}, 8 \mathrm{~V}$, $9 \mathrm{~V}$ and $10 \mathrm{~V}$ for probe $1,2,3$ and 4 respectively. The three probe currents collapsed at 00:31:13 UT, after which the currents immediately rebounded and then collapsed again. In this case, the currents on probe 1, 2 and 3 stay zero or close to zero until approximately 00:37, when probe 3 and 4 start to recover, while probes 1 and 2 stay zero or close to zero. During the short rebound just after 00:31:13, the probe currents maintain approximately the same distance on the $y$-axis. In the left panel of Fig.4, we see the output voltage across the solar cell arrays as NorSat-1 exits eclipse. As the satellite exits from and enters into eclipse, the solar cell array turn on and off respectively. The solar cell array voltage will start out after eclipse exit at around $23 \mathrm{~V}$, and decrease to between $16 \mathrm{~V}$ and $18 \mathrm{~V}$ as the satellite moves through the dayside, and the panels heat up. The material temperature then increases as the solar cell operation goes from peak power voltage to open-circuit voltage (as the battery charge approaches maximum). In Fig. 4 (left), the offvoltage is taken to be zero, assuming the individual circuits are grounded.

\section{RESULTS}

The events described in Fig. 4 are not unique. This current collapse happens on most eclipse exits. Furthermore, because of NorSat-1's stable orbit, all eclipse exit data is gathered from a fairly uniform part of the ionosphere, as seen in Fig. 3. This spatial uniformity provides ideal conditions for superposing the measured timeseries. Superposing a set of timeseries entails normalizing them to a fixed length, and then taking the mean of all the superposed timeseries. In Fig. 5, we show a superposition of 795 eclipse exit and 1050 eclipse entry events, in a long-term and expanded view perspective respectively. The shaded area behind the graphs indicates that the data was measured inside Earth's shadow. 

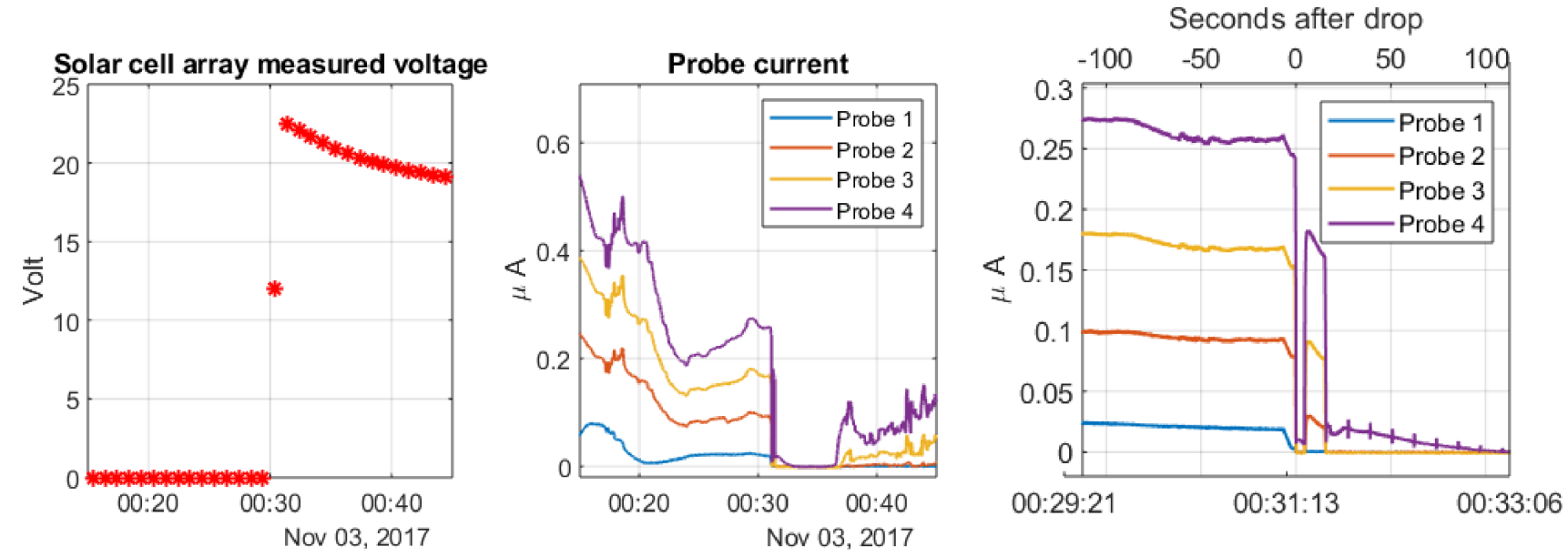

Fig. 4. The solar cell array output voltage (left panel), during a 30 minute interval of the date interpolated to a low-resolution grid (middle panel) and a full-resolution interval (right panel) showing the current collapse upon exiting eclipse on 3 November 2017 . The probe biases were 6 V, 8 V, 9 V and $10 \mathrm{~V}$ for probe 1, 2, 3 and 4, respectively. In the solar cell array voltage plot, a zero-voltage is assumed when the arrays are switched off; this rests on whether the individual solar cell circuits are grounded or not - if the circuits are not grounded the potential will float.

The point of eclipse was for each event found by adjusting the solar zenith angle for the satellite altitude. Upon eclipse exit, light that hit the solar cells have travelled extensively through Earth's dense atmosphere. This means the light can have undergone refraction and UV-attenuation. We adjusted for the introduced uncertainty by simply aligning each individual timeseries by the first sharp current drop. The average adjusted solar zenith angle at which the current collapsed was 90.5 degrees. In both figures, the top panels show the probe currents, which were biased at $6 \mathrm{~V}, 8 \mathrm{~V}, 9 \mathrm{~V}$ and $10 \mathrm{~V}$ for probe 1, 2, 3 and 4 respectively. In the bottom panels we see the calculated electron density, with black lines for the density based on all four probes, and red lines for the density as calculated for probes 2, 3 and 4 . The data is taken from NorSat-1's operation from 4 January to 8 May 2018. The data is then gathered in the southern hemisphere summer and autumn, or, consequently, the northern hemisphere winter and spring. On the upper $x$-axes of Fig. 5, we display the median magnetic latitude from which the data points were sampled. Here, we note that the large-scale peaks in the density observed on both sides of the solar terminator is caused by the equatorial ring current.

The general drop we observe in the measured density as the satellite crosses the eclipse boundary is physical, which we have confirmed by examining data from the International Reference Ionosphere (IRI)[27], [28]. During the eclipse exit events, the two density measures, one made using 4 probes and one made using the three highest biased probes, initially stay almost identical, and differ significantly after the eclipse exit. The lowest biased probe current (probe 1) stays zero or near-zero after the exit. For the eclipse entry events, no sudden increase in the measured density is seen, though a somewhat dramatic rise in currents is observed roughly 4 minutes after eclipse entry.

In panels e, f, g, and h of Fig. 5, we can see the detailed behavior of the current collapse. The probe currents in the eclipse exit events echo Fig. 4 closely, even though the eclipse exit data in Fig. 5 is the result of 795 events in superposition. The currents collapse initially, then immediately rebound, and then drop again. By 15 seconds, the second drop has occurred. As can be seen in the right panels of Figs. 5, in the case of eclipse entries, no sudden change is observed. There, the measured density stays fairly flat, with the red and black lines agreeing.

Satellite orientation is important to the operation of NorSat-1. The main solar cell array will not operate at peak power voltage when it is oriented away from the Sun. In addition, depending on the orientation, probes may be situated in the plasma wake caused by the moving satellite. Defining a Sun-alignment angle $\alpha$ as the angle between a vector normal to the main solar cell array and a vector connecting the spacecraft to the Sun, we can separate NorSat1 's eclipse exits into roughly two categories, schematically drawn in Fig. 6. In the majority of the exits, NorSat-1 orbits with the AIS instruments slightly tilted towards Earth (top). In $13 \%$ of the exits, the AIS instruments points straight towards Earth's surface while the main solar array is almost normal to the Sun-spacecraft vector (Fig. 6, bottom). Fig. 7 (top) shows the currents across all probes when NorSat-1 exits eclipse during these two configurations, where the angle $\alpha$ takes on values of $40^{\circ}<\alpha<90^{\circ}$ (left) and $\alpha<40^{\circ}$ (right). In the 140 non-aligned exits, the probe currents do not drop substantially upon eclipse exit. In 882 exits NorSat1 maintained an $\alpha$ angle less than $40^{\circ}$.

\section{DISCUSSION}

The observed current drop can be ascribed to a drop in the spacecraft potential. Since the probe biases are set relative to the spacecraft potential, a change in the spacecraft potential would also change each probe's bias against the plasma potential. A drop in the spacecraft potential would then cause all four probe current values to slide down the I-V curve in 

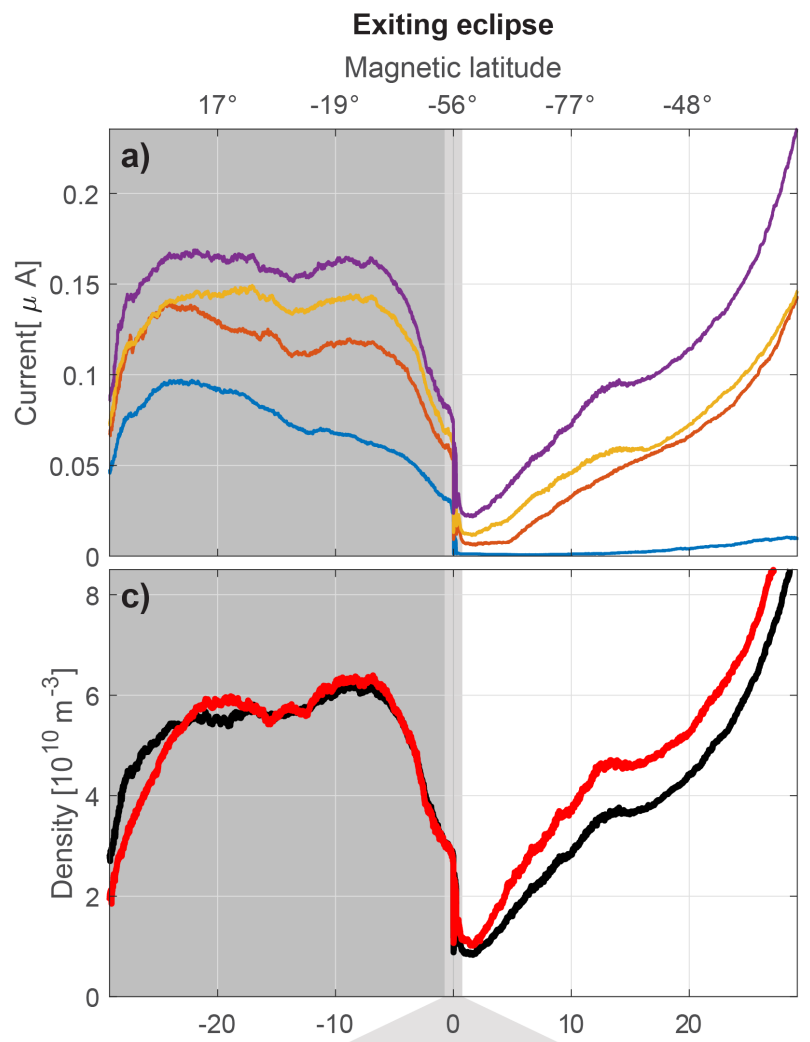

Minutes after exit
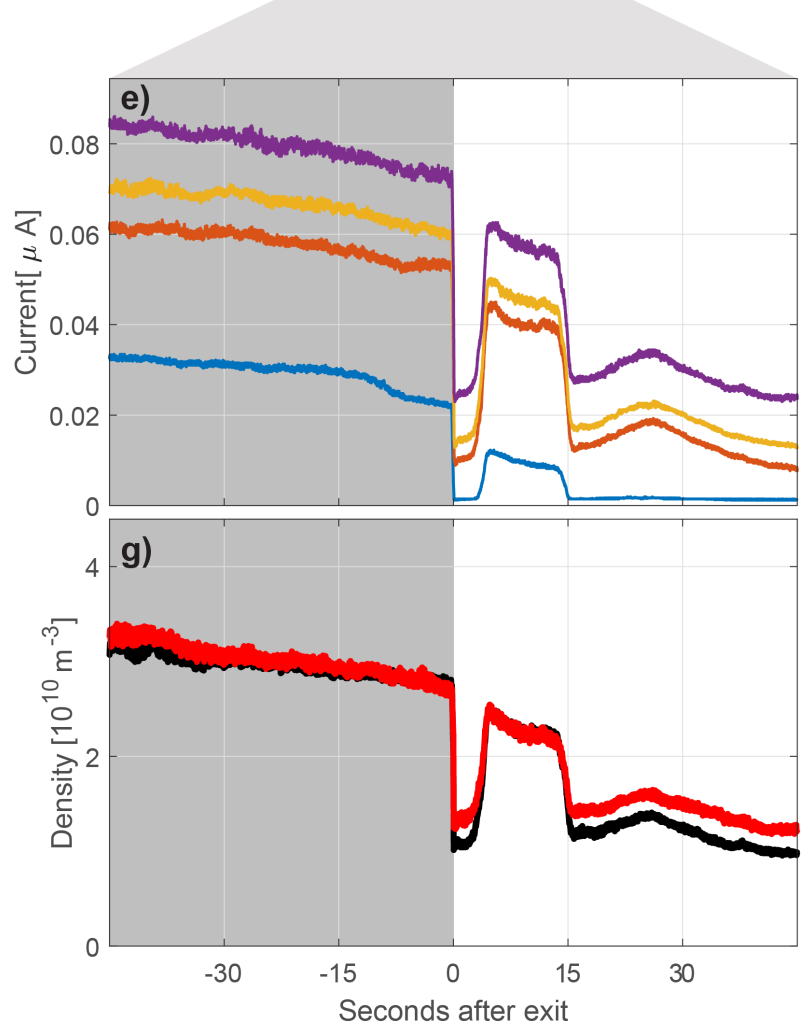

\section{Entering eclipse}

Magnetic latitude
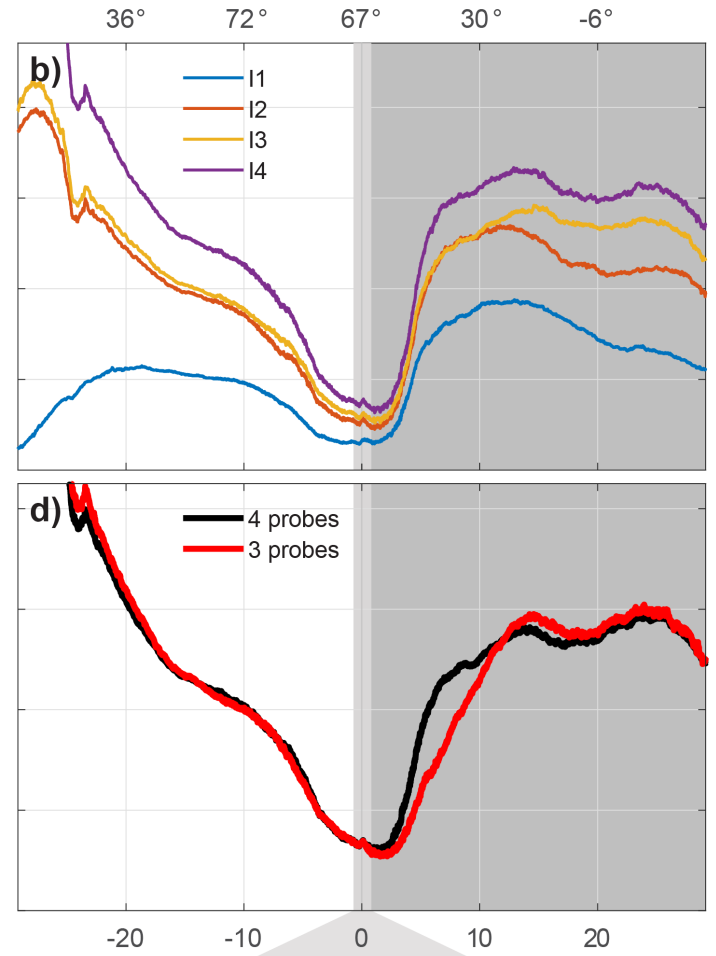

Minutes after entrence
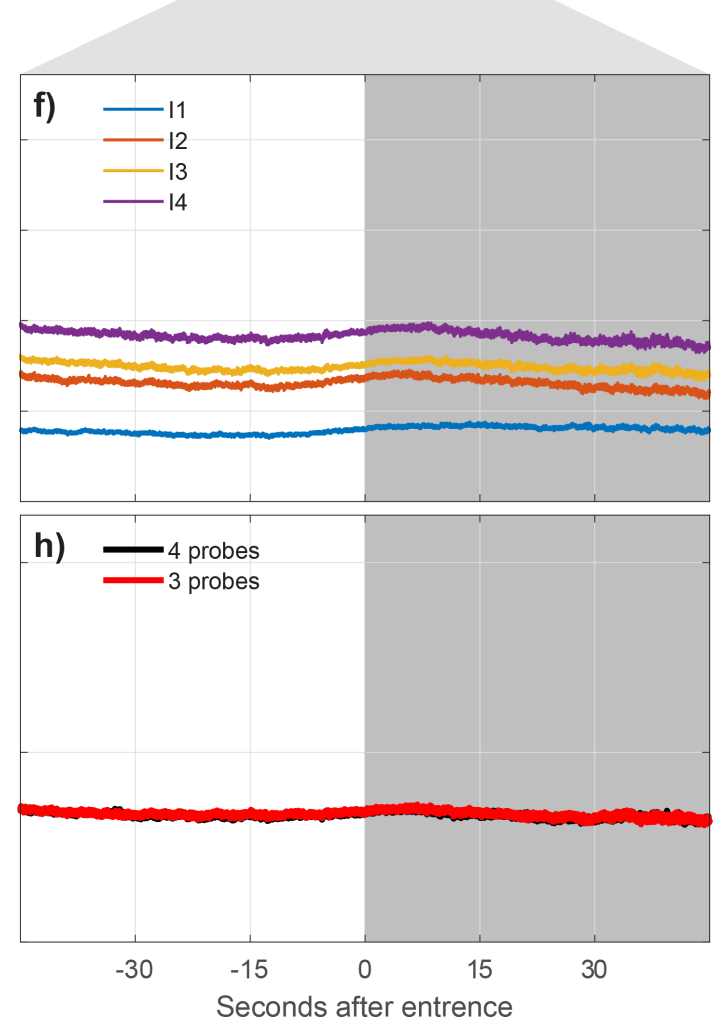

Fig. 5. The median of 795 exits and 1050 entries into eclipse in superposition. The four top panels show a long-term view of the terminator crossings, while the four bottom panels show a short-term view. The left panels (a, c, e, and g) show eclipse exits, and the right panels show entries (b, d, f, and h). In panels a b, e, and f, the four probe currents are displayed, while panels c, d, g, and h show the calculated density using Eq. 2, using probes 2,3 and 4 (red), and all probes (black). On the top $x$-axis, median magnetic latitude for the sampled data is shown. 
Fig. 2, and out of the electron saturation region.

Charging effects that are either continuous across Earth shadow eclipse or varying with respect to plasma conditions can be discounted, since a form of current drop is reliably occurring on every NorSat-1 eclipse exit. Photoemission is caused by incident sunlight on the spacecraft conductive surfaces, and is thus "switched on" upon eclipse exit. However, photoemission would cause an electron current away from the spacecraft, which would lead to a higher spacecraft potential, and no observable drop in the probe currents.

A possible explanation for the acute current drop upon eclipse exit displayed in Fig. 5 is spacecraft charging by solar cell array electron collection. The solar cell array of NorSat-1 contain small gaps between the individual solar cell units, and under sunlit conditions the cells are very positively biased with respect to the ambient plasma potential (see Fig. 8). A large current of electrons is then collected, thus lowering the spacecraft potential. Since the m-NLP probes are biased with respect to the spacecraft potential, the Langmuir probe current values then slide down the I-V curve of Fig. 2, and out of the electron saturation region. During eclipse, the cell units are in effect turned off, resulting in no current being collected.

A similar effect has been observed on the International Space Station (ISS) for many years. Coined "Rapid Charging Events", the solar cell charging induced drop in ISS spacecraft potential is described in [29], but is also extensively covered in [26], [30]-[34]. Examining ISS data, [31] analyze the measured spacecraft potential and propose that the high electron current to the solar cells causes rapid negative charging of the ISS. Statistically, [29] determined that these Rapid Charging Events only occurred in relatively low ambient plasma densites of $\propto 10^{10} \mathrm{~m}^{-3}$, a value that matches the polar and sub-polar ionospheric plasma density at the altitude of NorSat-1.

\section{Plasma wake and orientation}

Initially, in the 140 high- $\alpha$ exits shown in Fig. 7, the solar irradiance at the main solar array will be negligible, and so only the body-attached solar panels will operate at peak power voltage while NorSat-1 exits eclipse. This in turn keeps the probe currents from collapsing. However, as is seen in Fig. 9 (bottom) for the high- $\alpha$ exits, NorSat-1 gradually orients the main solar array against the Sun. By 4 minutes, the current through probe 1 has dropped to zero.

A separate but related matter is the case of highly expanded plasma sheaths [35], [36]. The plasma sheath around the solar cell arrays will upon eclipse exit expand due to the potential difference between the solar cells and the surrounding plasma. Since NorSat-1 is mainly orbiting with the mNLP system trailing the satellite in the southern hemisphere, one or more probes might be inside the electron-depleted plasma wake left by the solar cell arrays. Conversely, when NorSat-1 enters eclipse in the northern hemisphere, it usually orbits with the m-NLP system in the ram direction.

To further understand the effect of the expanded plasma wake, consider Fig. 7. For the 140 eclipse exits with

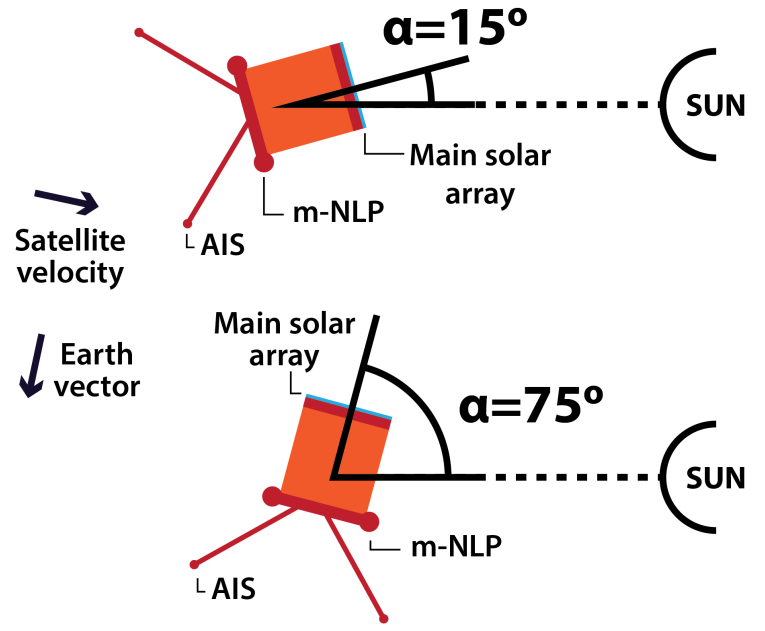

Fig. 6. Schematic drawing showing NorSat-1 orbiting in the southern hemisphere with $\alpha=15^{\circ}$ (top), and $\alpha=75^{\circ}$ (bottom), $\alpha$ being the angle between a vector normal to the main solar cell array and a vector connecting the spacecraft to the Sun. The vector labeled "Earth vector" points towards the center of Earth.
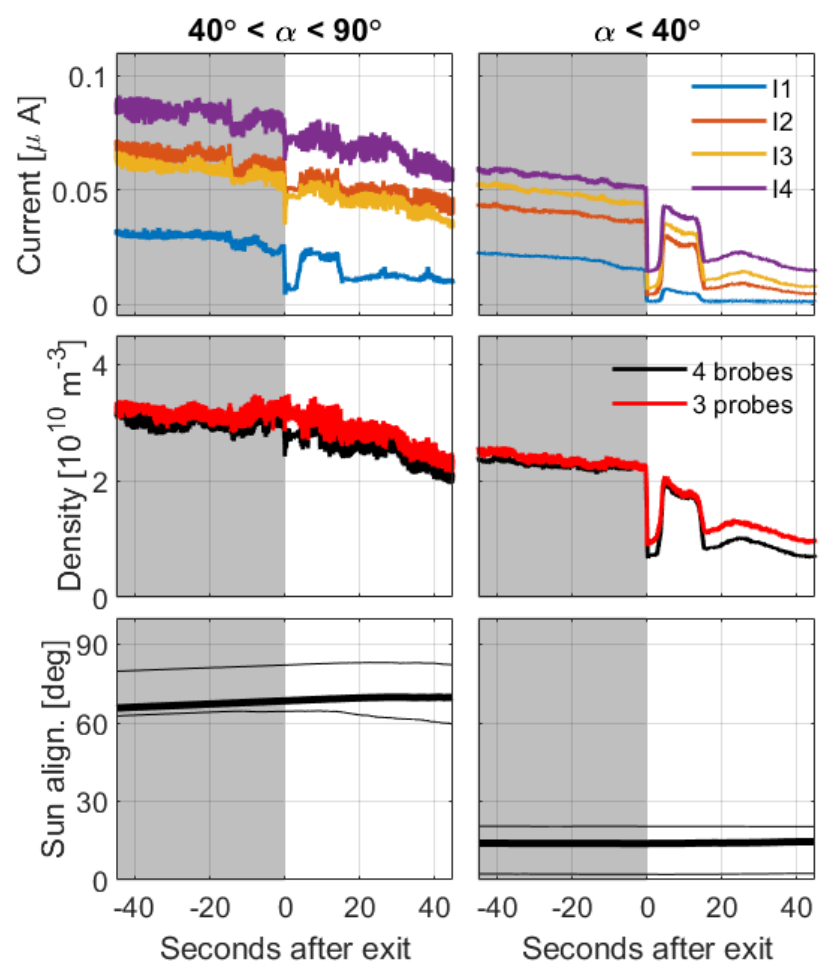

Fig. 7. Median of 140 eclipse exits where NorSat- 1 orients the main solar arrays at a large angle against the direction of the Sun (left) and 882 eclipse exits where the NorSat-1 main solar arrays roughly tracks the Sun (right). NorSat-1 was in otherwise normal operation during these exits. The bottom panels show the angle $\alpha$ as defined in the top schematic drawing. See Fig. 5 for a detailed plot explanation of the current and density data. 


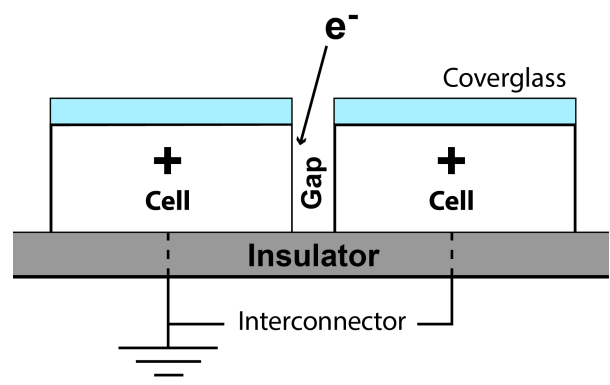

Fig. 8. A schematic figure of the solar cell units on NorSat-1, showing electron collection through the cell unit gaps.

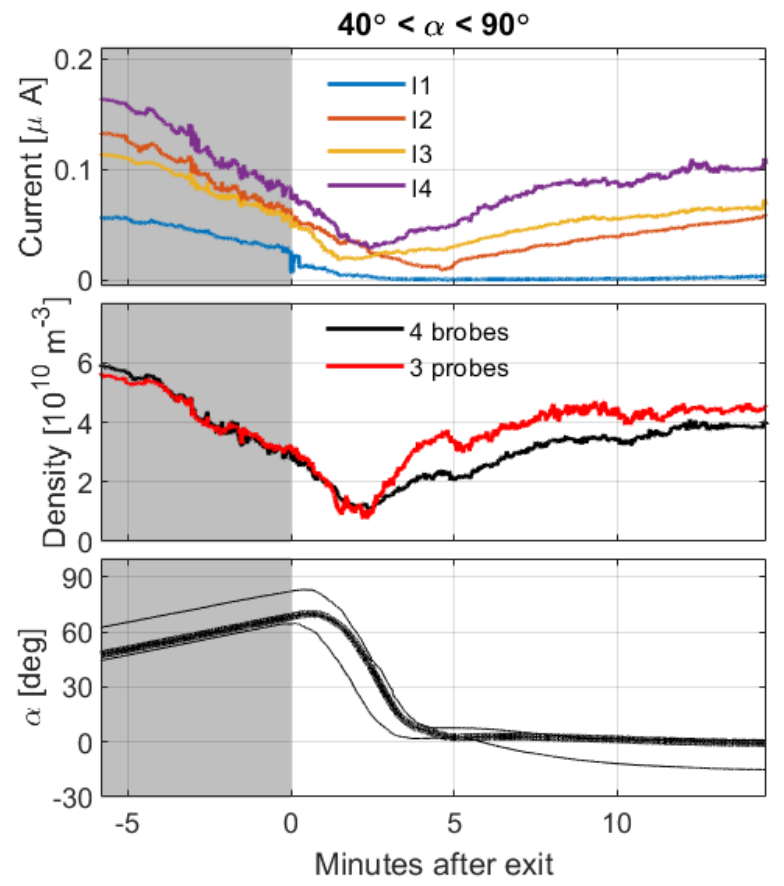

Fig. 9. A zoomed-out view of the 140 timeseries in which $40^{\circ}<\alpha<90^{\circ}$ upon eclipse exit (displayed in Fig. 7).

$40^{\circ}<\alpha<90^{\circ}$, two probes are situated inside the plasma wake, while two probes are kept outside. We can observe this both in the geometric schematic and the non-aligned probe current plot. In the latter, the current through probe 2 is consistently higher than the current through probe 3 , despite probe 2 being biased at $7 \mathrm{~V}$ and probe 3 being biased at 8 $\mathrm{V}$. This is clear evidence that the electron depleted plasma wake is affecting the probe current collection. In Fig. 9 we can observe that as NorSat-1 changes its orientation from non-alignment to Sun-tracking, the current through probe 3 gradually overtakes the current through probe 2 , indicating that all probes are situated within the satellite plasma wake.

\section{Current rebound}

At face value, the proposed solution does not offer an explanation into why the current rebounds and then recollapses after the initial collapse. This rebound effect might be explained by the proportion of solar cell circuits that are shut off, which is automatically handled by the on-board control systems. This computer-handled solar cell circuitry control has been found to influence the measured ISS solar cell charging at eclipse exit [33], [34], [37]. The timescale of the rebound effect observed in Fig. 5 might then be associated with a characteristic timescale of the onboard circuitry.

\section{Charging stabilization}

Electron attraction by solar cell array naturally persists throughout NorSat-1's dayside orbit. When the satellite exits eclipse, the solar cell array voltage quickly reaches a peak of about 23 V. During the orbit through Earth's dayside, the potential of the solar cell arrays gradually decreases to about $16 \mathrm{~V}$, resulting in the number of electrons collected drastically decreasing as NorSat-1 orbits through Earth's dayside. Additionally, as mentioned, the on-board control systems will automatically shut off individual solar cell circuits throughout the satellite's sunlit orbit. This will decrease the number of solar cell units capable of attracting electrons.

\section{CONCLUSION}

As NorSat-1 exits the shadow of Earth into sunlight in the southern hemisphere, the electron currents collected by the Langmuir probes of the m-NLP system collapse. The current collapse happens with a strong predictability on most orbits. This probe current drop causes a temporary disturbance in the measured electron density values.

Solar arrays can cause highly negative charging on the spacecraft platform under the sunlit condition, upon spacecraft eclipse exit [29]. On NorSat-1, negative electrodes of the on board solar arrays are connected to the spacecraft platform. Since there are small gaps between the solar cells, under sunlit conditions the cells are biased positively with respect to the ambient plasma potential thus collecting significant amounts of electrons. As a consequence, the NorSat1 spacecraft potential could be highly negatively charged, causing the m-NLP probe currents to enter the electron retardation regime. The current collapse is highly dependent on satellite orientation.

From a practical point of view, we recommend correcting the NorSat-1 data. Such a correction can be achieved by attaching a quality flag to the data product, or by taking into account the changes in I-V curve characteristics caused by a probe leaving the electron saturation region when calculating the m-NLP density estimate.

In the future, safe-guarding against the m-NLP probe current collapse is desirable. Such mitigation can be done by implementing ways to measure or control the spacecraft potential, for example by implementing a controlled electron emission mechanism. Other mitigation techniques include grouting, or filling in the solar cell unit gaps with a dielectric material [38], and connecting spacecraft electrical ground to the solar cell circuitry's positive end. 


\section{ACKNOWLEDGEMENTS}

This research has received financial support from Research Council of Norway project 275653, and ESA PRODEX project 4200090335. The authors would like to thank the Norwegian Space Center for selecting the m-NLP payload for NorSat-1 and for their financial support of the project. We would also like to show our gratitude to UTIAS-SFL for their support during the integration of the instrument in the satellite and to StatSat for putting the instrument into daily operations. The work is also a part of the 4DSpace initiative at the University of Oslo. The authors would also like to thank Alexander M. Beattie, Arne Pedersen, Joseph I. Minow, Grant Miars, Omar Leon, Kathryn Kirsch, and Noah Hood for helpful comments and discussions.

\section{REFERENCES}

[1] B. Walter, P.-L. Levesque, G. Kopp, B. Andersen, I. Beck, W. Finsterle, Manfred Gyo, K. Heuerman, S. Koller, N. Mingard, A. R. Oliva, D. Pfiffner, Ricco Soder, M. Spescha, M. Suter, and W. Schmutz, "The CLARA/NORSAT-1 solar absolute radiometer: instrument design, characterization and calibration," Metrologia, vol. 54, no. 5, p. 674, 2017. [Online]. Available: http://stacks.iop.org/0026$1394 / 54 / \mathrm{i}=5 / \mathrm{a}=674$

[2] K. S. Jacobsen, A. Pedersen, J. I. Moen, and T. A. Bekkeng, "A new Langmuir probe concept for rapid sampling of space plasma electron density," Measurement Science and Technology, vol. 21, no. 8, p. 085902, 2010. [Online]. Available: http://stacks.iop.org/0957$0233 / 21 / \mathrm{i}=8 / \mathrm{a}=085902$

[3] T. A. Bekkeng, K. S. Jacobsen, J. K. Bekkeng, A. Pedersen, T. Lindem, J.-P. Lebreton, and J. I. Moen, "Design of a multi-needle Langmuir probe system," Measurement Science and Technology, vol. 21, no. 8, p. 085903, 2010. [Online]. Available: http://stacks.iop.org/0957-0233/21/i=8/a=085903

[4] D. A. Lorentzen, J. Moen, K. Oksavik, F. Sigernes, Y. Saito, and M. G. Johnsen, "In situ measurement of a newly created polar cap patch," Journal of Geophysical Research: Space Physics, vol. 115, no. A12, 2010. [Online]. Available: https://agupubs.onlinelibrary.wiley.com/doi/abs/10.1029/2010JA015710

[5] J. Moen, K. Oksavik, T. Abe, M. Lester, Y. Saito, T. A. Bekkeng, and K. S. Jacobsen, "First in-situ measurements of HF radar echoing targets," Geophysical Research Letters, vol. 39, no. 7, 2012. [Online]. Available: https://agupubs.onlinelibrary.wiley.com/doi/abs/10.1029/2012GL051407

[6] K. Oksavik, J. Moen, M. Lester, T. A. Bekkeng, and J. K. Bekkeng, "In situ measurements of plasma irregularity growth in the cusp ionosphere," Journal of Geophysical Research: Space Physics, vol. 117, no. A11, 2012. [Online]. Available: https://agupubs.onlinelibrary.wiley.com/doi/abs/10.1029/2012JA017835

[7] K. A. Lynch, D. L. Hampton, M. Zettergren, T. A. Bekkeng, M. Conde, P. A. Fernandes, P. Horak, M. Lessard, R. Miceli, R. Michell, J. Moen, M. Nicolls, S. P. Powell, and M. Samara, "MICA sounding rocket observations of conductivity-gradient-generated auroral ionospheric responses: Small-scale structure with large-scale drivers," Journal of Geophysical Research: Space Physics, vol. 120, no. 11, pp. 9661-9682, Nov. 2015. [Online]. Available: https://agupubs.onlinelibrary.wiley.com/doi/abs/10.1002/2014JA020860

[8] A. Spicher, A. A. Ilyasov, W. J. Miloch, A. A. Chernyshov, L. B. N. Clausen, J. I. Moen, T. Abe, and Y. Saito, "Reverse flow events and small-scale effects in the cusp ionosphere," Journal of Geophysical Research: Space Physics, vol. 121 , no. 10 , pp. 10,466-10,480, Oct. 2016. [Online]. Available: https://agupubs.onlinelibrary.wiley.com/doi/abs/10.1002/2016JA022999

[9] T. A. Bekkeng, Helgeby, E. S., Pedersen, A., Trondsen, E., Lindem, T., and Moen, J. I., "Multi-needle Langmuir probe system for electron density measurements and active spacecraft potential control on cubesats (submitted)," IEEE Trans. Aerosp. Electron. Syst., 2017.

[10] H. M. Mott-Smith and I. Langmuir, "The Theory of Collectors in Gaseous Discharges," Physical Review, vol. 28, no. 4, pp. 727-763, Oct. 1926. [Online]. Available: https://link.aps.org/doi/10.1103/PhysRev.28.727

[11] F. F. Chen, "Langmuir Probe Diagnostics," IEEE -ICOPS meeting, Jeju, Korea, June 5, 2003, 2003.

[12] L. Conde, "An introduction to Langmuir probe diagnostics of plasmas," Madrid: Dept. FÃsica. ETSI AeronÃ ;ut ngenieros AeronÃ ;uticos Universidad PolitÃ_C cnica de Madrid, pp. 1-28, 2011.
[13] J. P. Lebreton, S. Stverak, P. Travnicek, M. Maksimovic, D. Klinge, S. Merikallio, D. Lagoutte, B. Poirier, P. L. Blelly, Z. Kozacek, and M. Salaquarda, "The ISL Langmuir probe experiment processing onboard DEMETER: Scientific objectives, description and first results," Planetary and Space Science, vol. 54, no. 5, pp. 472-486, Apr. 2006. [Online]. Available: http://www.sciencedirect.com/science/article/pii/S0032063305002084

[14] T. A. Bekkeng, A. Barjatya, U.-P. Hoppe, A. Pedersen, J. I Moen, M. Friedrich, and M. Rapp, "Payload charging events in the mesosphere and their impact on Langmuir type electric probes," Ann. Geophys., vol. 31, no. 2, pp. 187-196, Feb. 2013. [Online]. Available: https://www.ann-geophys.net/31/187/2013/

[15] H. Hoang, L. B. N. Clausen, K. RÃ sed, T. A. Bekkeng, E. Trondsen, B. Lybekk, H. StrÃ $s$, D. M. Bang-Hauge, A. Pedersen, A. Spicher, and J. I. Moen, "The Multi-Needle Langmuir Probe System on Board NorSat-1," Space Science Reviews, vol. 214, no. 4, p. 75, Jun. 2018. [Online]. Available: https://link.springer.com/article/10.1007/s11214018-0509-2

[16] J. E. Allen, "Probe theory - the orbital motion approach," Physica Scripta, vol. 45, no. 5, p. 497, 1992. [Online]. Available: http://stacks.iop.org/1402-4896/45/i=5/a=013

[17] I. D. Sudit and R. C. Woods, "A study of the accuracy of various Langmuir probe theories," Journal of Applied Physics, vol. 76, no. 8, pp. 4488-4498, Oct. 1994. [Online]. Available: https://aip.scitation.org/doi/10.1063/1.357280

[18] A. R. Hoskinson and N. Hershkowitz, "Effect of finite length on the current voltage characteristic of a cylindrical Langmuir probe in a multidipole plasma chamber," Plasma Sources Science and Technology, vol. 15, no. 1, p. 85, 2006. [Online]. Available: http://stacks.iop.org/0963-0252/15/i=1/a=013

[19] H. Hoang, K. RÃ ed, T. A. Bekkeng, J. I. Moen, A. Spicher, L. B. N. Clausen, W. J. Miloch, E. Trondsen, and A. Pedersen, "A study of data analysis techniques for the multi-needle Langmuir probe," Measurement Science and Technology, vol. 29, no. 6, p. 065906, 2018.

[20] S. T. Lai, "A critical overview on spacecraft charging mitigation methods," IEEE Transactions on Plasma Science, vol. 31, no. 6, pp. 1118-1124, Dec. 2003

[21] T. Mikaelian, "Spacecraft Charging and Hazards to Electronics in Space,” arXiv:0906.3884 [physics], Jun. 2009, arXiv: 0906.3884 [Online]. Available: http://arxiv.org/abs/0906.3884

[22] M. U. Siddiqui, L. E. Gayetsky, M. R. Mella, K. A. Lynch, and M. R. Lessard, "A laboratory experiment to examine the effect of auroral beams on spacecraft charging in the ionosphere," Physics of Plasmas, vol. 18, no. 9, p. 092905, Sep. 2011. [Online]. Available: https://aip.scitation.org/doi/10.1063/1.3640512

[23] D. FERGUSON, "The voltage threshold for arcing for solar cells in Leo - Flight andground test results," in 24th Aerospace Sciences Meeting. American Institute of Aeronautics and Astronautics, 1986 [Online]. Available: https://arc.aiaa.org/doi/abs/10.2514/6.1986-362

[24] I. Katz, V. Davis, and D. Snyder, "Mechanism for spacecraft charging initiated destruction of solar arrays in GEO," in 36th AIAA Aerospace Sciences Meeting and Exhibit. American Institute of Aeronautics and Astronautics, 1998. [Online]. Available: https://arc.aiaa.org/doi/abs/10.2514/6.1998-1002

[25] I. O. for Standardization, ISO 11221: Space systems - Space solar panels - Spacecraft charging induced electrostatic discharge test methods. International Organization for Standardization, Aug. 2011.

[26] J. Huang, L. Guoqing, and J. Lixiang, "LEO Spacecraft Charging and Discharging Induced by High Voltage Solar Arrays," Journal of Astrophysics \& Aerospace Technology, vol. 3, no. 1, 2015. [Online] Available: https://www.omicsonline.org/open-access/leo-spacecraftcharging-and-discharging-induced-by-high-voltage-solar-arrays-23296542-1000111.php?aid=55844

[27] D. Bilitza and B. W. Reinisch, "International Reference Ionosphere 2007: Improvements and new parameters," Advances in Space Research, vol. 42, no. 4, pp. 599-609, Aug. 2008. [Online]. Available: http://www.sciencedirect.com/science/article/pii/S0273117708000288

[28] D. Bilitza, D. Altadill, Y. Zhang, C. Mertens, V. Truhlik, P. Richards, L.-A. McKinnell, and B. Reinisch, "The International Reference Ionosphere 2012 a model of international collaboration," Journal of Space Weather and Space Climate, vol. 4, p. A07, 2014. [Online]. Available: https://www.swscjournal.org/articles/swsc/abs/2014/01/swsc130043/swsc130043.html

[29] P. Craven, K. Wright, J. Minow, V. Coffey, T. Schneider, J. Vaughn, D. Ferguson, and L. Parker, "Survey of International 
Space Station Charging Events," in 47th AIAA Aerospace Sciences Meeting including The New Horizons Forum and Aerospace Exposition, ser. Aerospace Sciences Meetings. American Institute of Aeronautics and Astronautics, Jan. 2009. [Online]. Available: https://arc.aiaa.org/doi/10.2514/6.2009-119

[30] H. M. Barsamian, "ISS Plasma Interaction: Measurements and Modeling," Mar. 2004. [Online]. Available: https://ntrs.nasa.gov/search.jsp?R=20040111048

[31] D. Ferguson, P. Craven, J. Minow, and K. Wright, "A Theory for Rapid Charging Events on the International Space Station," in 1st AIAA Atmospheric and Space Environments Conference, ser. Fluid Dynamics and Co-located Conferences. American Institute of Aeronautics and Astronautics, Jun. 2009. [Online]. Available: https://arc.aiaa.org/doi/10.2514/6.2009-3523

[32] J. Huang, Z. Yi, H. Zhao, L. Meng, and Y. Liu, "Mechanism for Rapid Charging Events on International Space Station," Journal of Spacecraft and Rockets, vol. 51, no. 3, pp. 917-921, 2014. [Online]. Available: https://doi.org/10.2514/1.A32702

[33] E. Willis, J. I. Minow, L. N. Parker, M. Z. Pour, C. Swenson, K.-i. Nishikawa, and L. Habash Krause, "Observations of Transient ISS Floating Potential Variations during High Voltage Solar Array Operations," in AIAA SPACE 2016, ser. AIAA SPACE Forum.
American Institute of Aeronautics and Astronautics, Sep. 2016 [Online]. Available: https://arc.aiaa.org/doi/10.2514/6.2016-5382

[34] E. M. Willis and M. Z. A. Pour, "A New Model for Plasma Interactions With High-Voltage Solar Arrays on the International Space Station," IEEE Transactions on Plasma Science, vol. 46, no. 4, pp. 1018-1026, Apr. 2018.

[35] S. B. Wang and A. E. Wendt, "Sheath thickness evaluation for collisionless or weakly collisional bounded plasmas," IEEE Transactions on Plasma Science, vol. 27, no. 5, pp. 1358-1365, Oct. 1999.

[36] B. Scheiner, S. D. Baalrud, B. T. Yee, M. M. Hopkins, and E. V. Barnat, "Theory of the electron sheath and presheath," Physics of Plasmas, vol. 22, no. 12, p. 123520, Dec. 2015. [Online]. Available: https://aip.scitation.org/doi/abs/10.1063/1.4939024

[37] J. I. Minow, I. Katz, P. D. Craven, V. A. Davis, B. M. Gardner, T. W. Kerslake, M. J. Mandell, L. N. Parker, T. J. Peshek, E. M. Willis, and K. H. Wright, "Evidence for Arcing on the International Space Station Solar Arrays," Proceedings of the 15th Spacecraft Charging Technology Conference, Jun. 2018.

[38] M. Ranjan, N. K. P. Vaghela, S. Mukherjee, M. Sankaran, and S. E. Puthanveettil, "Arc Mitigation via Solar Panel Grouting and Curing Under Simulated LEO-Like Plasma Environment," IEEE Transactions on Plasma Science, vol. 41, no. 12, pp. 3323-3328, Dec. 2013. 